\title{
THE EVOLUTION OF THE STRUCTURE AND MECHANICAL PROPERTIES OF ALUMINUM DURING ACCUMULATIVE ROLL BONDING
}

\begin{abstract}
K. V. Ivanov ${ }^{1}$ and E. V. Kudryavtsev ${ }^{2}$
UDC 669.71:539.24’25

The dependence of microstructure, microhardness, and strength properties in tensile strain tests of commercially pure aluminum on strain during accumulative roll-bonding was studied. The methods of transmission electron microscopy and electron back scattered diffraction patterns analysis were used to establish that a lamellar ultrafine structure is formed in aluminum after accumulative roll bonding. It is shown that aluminum microhardness, ultimate strength, and yield strength increase monotonically with the degree of true logarithmic strain increasing from 3.2 to 8.0. The reasons for the difference in the structure and mechanical properties of the aluminum studied from the ones relevant for the aluminum deformed by equalchannel angular pressing are discussed.
\end{abstract}

Keywords: aluminum, accumulative roll bonding, microstructure, mechanical properties.

\section{INTRODUCTION}

Ultrafine-grained materials obtained by severe plastic deformation (SPD), have unusual mechanical and functional properties attractive for practical application $[1,2]$. The most common methods of SPD are Equal-Channel Angular Pressing [3], Multiple Forging [4], and High-Pressure Torsion [5]. These methods are laborious, complex, and expensive from a technological point of view. In this regard, a search for new methods of SPD is carried out in Russia and abroad. Presently, Accumulative Roll Bonding (ARB), first suggested in [6], is one of the most attractive and rapidly developing methods to obtain ultrafine-grained structure by SPD. It has a number of technological and practical advantages over the other SPD methods: this method makes it possible to form composites with a given geometry of the strengthening phase distribution; it can be used in industry without significant updating of the core process equipment (rolling mills); the products are obtained as standard shape sheets and tapes. It is natural to expect that these advantages will make rolling by the abovementioned method common to produce materials with improved properties in the nearest future. Therefore the regularities of the ultrafine-grained structure and mechanical properties formation during multiple rolling are of significant interest. However the papers devoted to improving the properties of metals and alloys by the formation of ultrafine-grained structure using accumulative roll bonding are extremely scarce in Russian literature. The authors are familiar with the only Russian work related to this topic [7].

Hence the objective of this work was to study the microstructure forming during the metal deformation by accumulative roll bonding of different degrees, as well as to establish the relation between the structural characteristics and mechanical properties manifested by the material after the exposure mentioned.

${ }^{1}$ Institute of Strength Physics and Materials Science of the Siberian Branch of the Russian Academy of Sciences, Tomsk, Russia, e-mail: ikv@ispms.tsc.ru; ${ }^{2}$ Belgorod State National Research University, Belgorod, Russia, e-mail: kudryavtsev@bsu.edu.ru. Translated from Izvestiya Vysshikh Uchebnykh Zavedenii, Fizika, No. 1, pp. 140145, January, 2017. Original article submitted July 29, 2016; revision submitted December 2, 2016. 
TABLE 1. Chemical Composition of the Studied Aluminum, wt $\%$

\begin{tabular}{l|c|c|c|c|c|c|c|c|c}
\hline \hline \multicolumn{1}{c|}{ Elements } & $\mathrm{Al}$ & $\mathrm{Si}$ & $\mathrm{Fe}$ & $\mathrm{Cu}$ & $\mathrm{Mn}$ & $\mathrm{Mg}$ & $\mathrm{Zn}$ & $\mathrm{Ga}$ & $\mathrm{Ti}$ \\
\hline Experiment & 99.70 & 0.25 & 0.02 & 0.01 & 0.01 & - & - & - & 0.01 \\
\hline $\begin{array}{l}\text { State Standard } \\
\text { GOST 11069-2001 }\end{array}$ & $<99.70$ & $>0.15$ & $>0.16$ & $>0.01$ & $>0.03$ & $>0.02$ & $>0.04$ & $>0.03$ & $>0.01$ \\
\hline
\end{tabular}

\section{MATERIALS AND EXPERIMENTAL TECHNIQUES}

Aluminum A1070 was studied. Table 1 shows its chemical composition according to the State Standard GOST 11069-2001 and the measurement by X-ray microanalysis using a wave spectrometer. Prior to rolling, the material underwent recrystallization annealing at $500^{\circ} \mathrm{C}$ for 1 hour. Then 2 tapes of the material $1 \mathrm{~mm}$ thick, $30 \mathrm{~mm}$ wide, and $300 \mathrm{~mm}$ long were washed in acetone, treated with a metal brush through the surface, gathered in a "pack", fastened with a thin wire at the ends, and rolled at room temperature by $50 \%$ at the rolling mill VEM-3 at a speed of $0.1 \mathrm{~m} / \mathrm{sec}$. The diameter of the mill rolls was $80 \mathrm{~mm}$. The tape obtained was cut into halves lengthwise. 1 cycle of accumulative roll bonding was comprised of this sequence. 3 material samples were obtained after 4, 7, and 10 rolling cycles. The degree of true accumulated strain was $3.2,5.6$, and 8.0 respectively.

The structure was studied by transmission electron microscopy (TEM) and electron backscattered diffraction (EBSD) patterns analysis in the plane containing the rolling direction and the normal direction. The foils for TEM were produced by ion slicing at the accelerating voltage of $6 \mathrm{kV}$ and the ion beam incident angle of $4^{\circ}$ using EM-09100IS Ion Slicer (Jeol). The same samples were used for EBSD analysis. Transmission analysis was performed at the accelerating voltage of $200 \mathrm{kV}$ using JEM-2100 microscope. EBSD analysis was implemented at Pegasus system integrated with the scanning electron microscope Nova Nanolab provided with a field emission gun. The data were obtained at the accelerating voltage of $20 \mathrm{kV}$ and the working distance of $7 \mathrm{~mm}$. The size of the scanned areas was $25 \times 14 \mu \mathrm{m}$ with the step size of $0.1 \mu \mathrm{m}$. The Kikuchi-patterns formed by the backscattered electrons were automatically indexed by "TSL OIM data collection" software (OIM - Orientation Imaging Microscopy). The data pools were processed by the "TSL OIM analysis" software. When analyzing the data, it was assumed that the subgrain is the region, within which the grain-boundary angle of the crystal lattice in the two neighboring scanning points was less than $2^{\circ}$. For the grains, this angle was considered less than $15^{\circ}$. The diameter of the circle, which area was equal to the grain (subgrain) area was taken as the grain (subgrain) size. The average size was calculated by the formula

$$
<d>=\frac{\sum_{i=1}^{N} S_{i} d_{i}}{\sum_{i=1}^{N} S_{i}}
$$

where $S_{i}$ and $d_{i}$ are the area and the size of the $i$-grain respectively.

The Vickers microhardness was measured by Duramin- 5 at the indentor load of $0.25 \mathrm{~N}$ for $15 \mathrm{~s}$.

The tension of samples with $20 \times 6.5 \times 1 \mathrm{~mm}$ test section was carried out at the INSTRON 3369 test machine at room temperature and the speed of $1 \cdot 10^{-4} \mathrm{~s}^{-1}$.

\section{RESULTS AND DISCUSSION}

TEM analysis of the aluminum after four rolling cycles revealed the formation of a developed lamellar structure elongated in its rolling direction (Fig. 1a). The lamellae boundaries have a wide range of the grain-boundary angles-from a few tenths of a degree to large angles (more than $15^{\circ}$ ). The distance between lamellae boundaries 

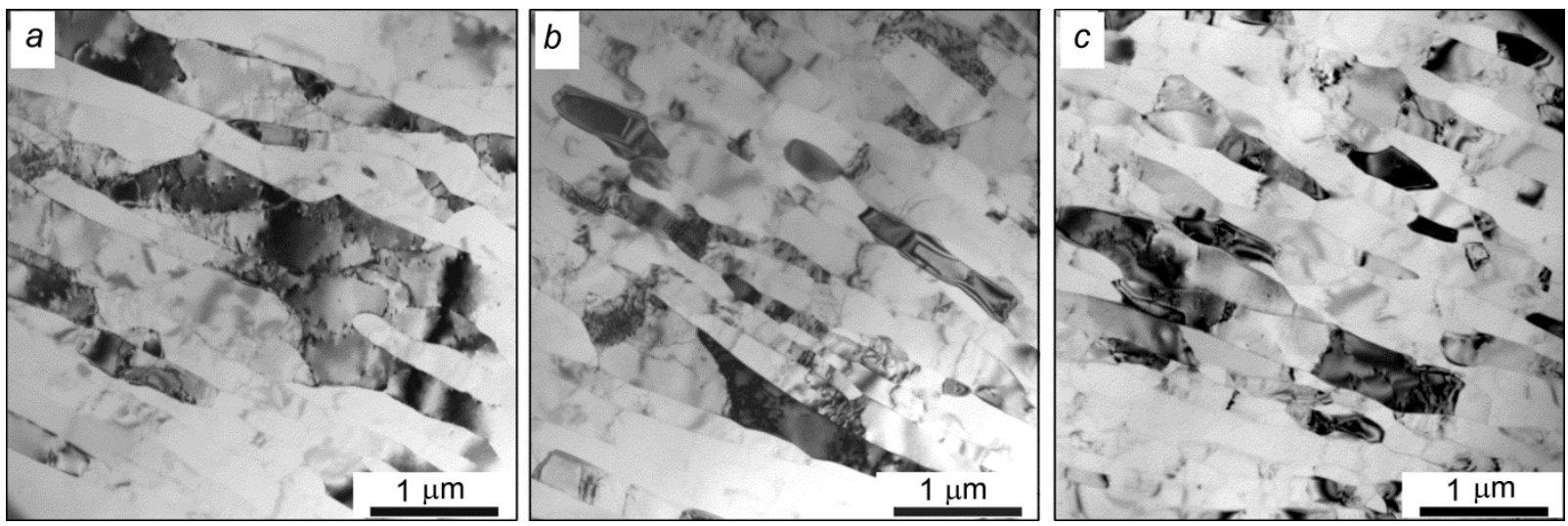

Fig. 1. Aluminum structure image after rolling: $a-4, b-7$, and $c-10$ cycles.

ranges $0.25-0.60 \mu \mathrm{m}$. The intralamellar structure is subdivided into fragments by the boundaries oriented at different angles to the rolling direction. The most of these fragments' boundaries are dislocation subboundaries with several degrees disorientation. Within these fragments, dislocation clusters in the form of nets and walls and single dislocations are observed.

The increase in the number of rolling cycles to seven resulted in the decrease of the distance between lamellae boundaries to $0.15-0.20 \mu \mathrm{m}$ in some regions (Fig. 1b). In other regions this distance remained within the $0.25-0.60 \mu \mathrm{m}$ range. Most of the boundaries separating fragments in the lamellae became perpendicular to the lamellae boundaries. There appeared a small number of fragments completely surrounded by high-angle boundaries, i.e. grains. The absence of dislocation structure within the grains and their shape — close to equiaxed—indicate that they were formed as a result of dynamic recrystallization during rolling. The dislocation structure within other fragments comprising the bulk of the material did not change in comparison with the structure after four rolling cycles.

The main differences in the aluminum structure after ten rolling cycles (Fig. 1c) from the structures considered above were as follows: first, there were almost no regions with the distance between lamellae boundaries exceeding $0.30 \mu \mathrm{m}$. Second, anglular disorientation of crystallites at the lamellae boundaries increased. Third, anglular disorientation at the boundaries of intralamellar fragments increased up to $7-10^{\circ}$, i.e. the structure elongation decreased. Fourth, the number of grains formed as a result of dynamic recrystallization increased.

Figures $2 a-c$ show grain-boundary angle profiles of the neighboring scanning points along the line in the direction normal to the rolling one obtained by EBSD analysis of the material after different number of rolling cycles. It is evident that there are two types of regions in the structure after four cycles: 1) with a large distance between the lamellae boundaries with high-angle disorientation (type 1, the $B C$ region of the profile in Fig. 2a), and 2) with relatively small (type 2, the $A B$ and $C D$ regions in Fig. $2 a$ ). In type 1 region occupying $2 / 3$ of the profile length, the distance between high-angle lamellae boundaries can reach $5 \mu \mathrm{m}$, while in type 2 regions it ranges from 0.4 to $1.0 \mu \mathrm{m}$. In the material that underwent seven rolling cycles, there is a decrease of the fraction of type 1 regions to $40 \%$ of the profile length (the $F G$ and $H I$ regions in Fig. $2 b$ ) and a decrease of the maximum distance between high-angle lamellae boundaries to $2.5-3.0 \mu \mathrm{m}$. In type 2 regions, the distance between lamellae boundaries with high-angle disorientation decreases to $0.3-0.8 \mu \mathrm{m}$. The increase in the number of rolling cycles to ten makes the difference between the regions of type 1 and 2 disappear due to the decrease of the distance between high-angle lamellae boundaries to $0.7 \mu \mathrm{m}$ and less in type 1 regions (the $J K$ region of the profile in Fig. $2 c$ ). The minimum distance between high-angle lamellae boundaries in the profile is $0.15 \mu \mathrm{m}$, and the average value is $0.40 \mu \mathrm{m}$. The result obtained is in satisfactory agreement with the literature data. For instance, in [8] it was shown by TEM that the distance between lamellae in $99.0 \%$ pure aluminum decreases to $0.24 \mu \mathrm{m}$ after eight cycles of accumulative roll bonding. In case of $99.5 \%$ pure aluminum, this value was $0.32 \mu \mathrm{m}$ after eight rolling cycles [9]. The discrepancy between the absolute values of the distance between lamellae boundaries obtained in this work and the ones mentioned above can be explained by the difference in the 
TABLE 2. Aluminum Structure Parameters after Accumulative Roll-Bonding

\begin{tabular}{c|c|c|c}
\hline $\begin{array}{c}\text { Rolling Cycles } \\
\text { Number }\end{array}$ & $\begin{array}{c}\text { Average Subgrain } \\
\text { Size, } \mu \mathrm{m}\end{array}$ & $\begin{array}{c}\text { Average Grain Size, } \\
\mu \mathrm{m}\end{array}$ & $\begin{array}{c}\text { High-Angle Grain } \\
\text { Boundaries Fraction, \% }\end{array}$ \\
\hline 4 & 2.0 & 6.9 & 56 \\
\hline 7 & 1.7 & 6.7 & 55 \\
\hline 10 & 1.0 & 1.7 & 68 \\
\hline
\end{tabular}
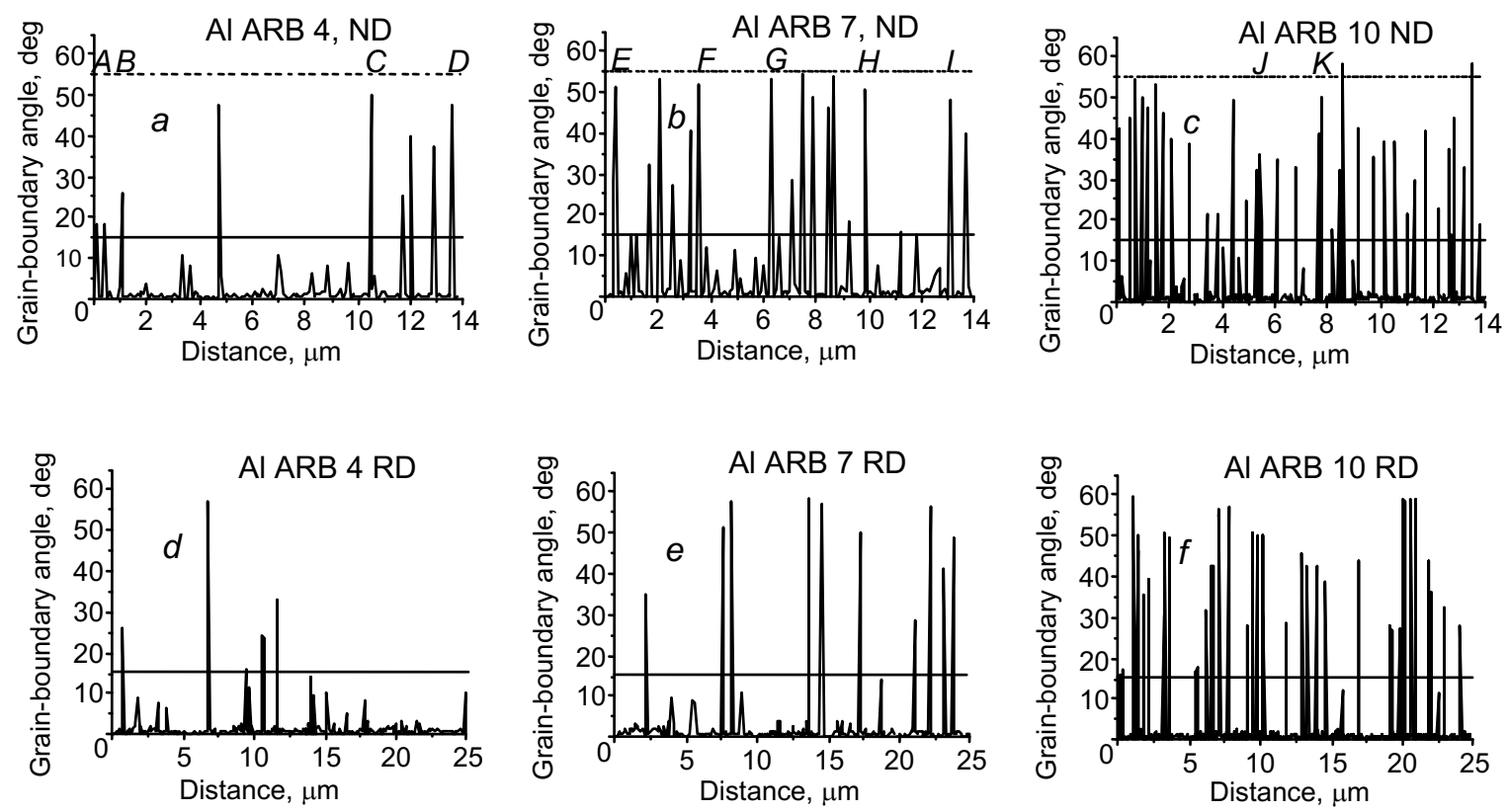

Fig. 2. The grain-boundary angle profile of aluminum crystal lattice after rolling obtained by EBSD analysis: $a-c-$ in the direction normal to the rolling one, $d-f-$ in the rolling direction, $a$, $d-4, b, e-7, c, f-10$ cycles. The horizontal line divides low- and high-angle disorientation.

purity of the aluminum analyzed. It is known that the purer the material is, the harder it is to refine its structure by severe plastic deformation [1].

The analysis of the grain-boundary angle profiles of the neighboring scanning points along the rolling direction (Fig. 2d-f) showed that the increase in the number of rolling cycles leads to the increase in the grain-boundary angle at high-angle boundaries perpendicular to the lamellae boundaries, and the decrease of the distance between them. Moreover, the distance decrease is most effective when there is a considerable plastic deformation achieved, i.e. when the number of rolling cycles exceeds seven.

Table 2 presents quantitative data on the average grain and subgrain sizes and the fraction of the high-angle grain boundaries in the structure of aluminum deformed to different degrees. It can be seen that there is mainly the evolution in the subgrain structure with the average subgrain size decrease by 1.2 times in case of the deformation with the rolling cycles number ranging from 4 to 7 . At that, the average grain size and the fraction of high-angle grain boundaries remain almost unchanged. More significant decrease (by 1.7 times) of the average subgrain size can be seen with the increase in the rolling cycles number up to ten. In this case, the grain structure is refined more effectively as well: the average grain size decreases by 4 times, and the fraction of high-angle grain boundaries reaches $\sim 70 \%$. It was earlier shown that $70 \%$ of high-angle grain boundaries fraction is characteristic of the ultrafine-grained aluminum (99.99\%) deformed by equal-channel angular pressing to the degree of true logarithmic strain $\sim 8[10,11]$. At the same 


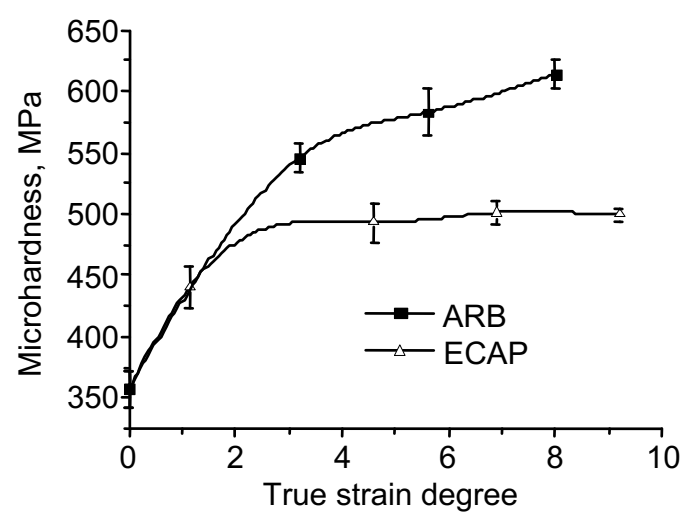

Fig. 3. Aluminum Microhardness Dependence on the Deformation Degree during Accumulative Roll Bonding (ARB) and Equal-Channel Angular Pressing (ECAP).

time according to [12], the fraction of high-angle grain boundaries determined by EBSD analysis in less pure aluminum $(99.0 \%)$ is $79 \%$ after six cycles of accumulative roll bonding.

Aluminum microhardness dependence on the deformation degree during accumulative roll bonding is given in Fig. 3. Similar dependence for the material after equal-channel angular pressing is given in the same Figure. It should be noted that, first, aluminum microhardness after rolling significantly exceeds aluminum microhardness after equalchannel angular pressing at the same degrees of the strain applied. The reason for this phenomenon may be the fact that sufficiently large number of boundaries is formed from the outer surface during rolling. Aluminum is a reactive metal, and its surface is always covered with a thin oxide film. During bonding with other external surface in the rolling process, a high-angle boundary is formed with a high content of impurities in it. These impurities may have a retarding effect on the dislocations motion and change the properties of the boundary itself, for example, reduce its ability to migrate or restrain grain boundary sliding. The second thing is that during equal-channel angular pressing, the microhardness stops to grow after several passes and further increase in the deformation degree has almost no influence on it. In case of rolling, the microhardness grows up to the degree of true deformation 8.0. At the same time, there are data that the values of microhardness and tensile strength in $99.5 \%$ pure aluminum stop to grow after six rolling cycles (the degree of true logarithmic deformation 4.8) [9]. It can be assumed that this contradiction is related to the purity of the aluminum used, however, further confirmation is needed.

Figure 4 shows engineering flow curves for aluminum samples after different quantity of accumulative roll bonding cycles. It can be seen that the stage of strain hardening reaches $2-3 \%$ of the deformation in the aluminum after rolling, which is higher than in the aluminum deformed using other SPD schemes. For example, in aluminum (99.99\%) deformed by ECAP to the strain degree of $\sim 8$ the stage of strain hardening lasts less than $1 \%$ of the deformation at the same strain rate [13]. The ability to preserve strain hardening is a positive property, indicating that the material preserves the resistance to the deformation localization. Aluminum ultimate strength and yield strength (Fig. 5) increase monotonically with increasing degree of strain during rolling, and the plasticity (considered as the deformation to fracture during tension) decreases sharply after the first rolling cycles with almost no further change. The growth of strength properties without reducing the deformation to fracture is also a positive property for the material used for practical construction purposes.

\section{CONCLUSIONS}

1. A lamellar ultrafine structure is formed in aluminum of commercial purity after accumulative roll bonding. The structure evolution during accumulative roll bonding occurring with increasing rolling cycles number manifests as the decrease of the distance between the lamellae boundaries and the respective perpendicular boundaries, and the 


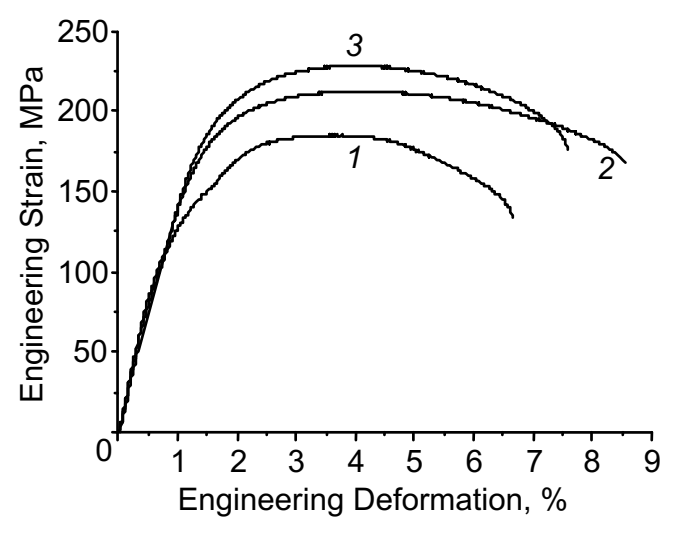

Fig. 4

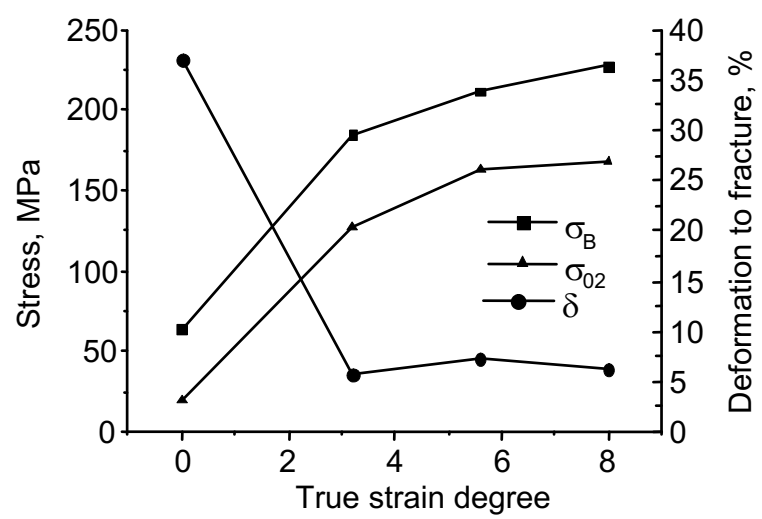

Fig. 5

Fig. 4. Aluminum Flow Curves after 4 (cur. 1), 7 (cur. 2), and 10 (cur. 3) Rolling Cycles.

Fig. 5. Dependences of Aluminum Ultimate Strength, $\left(\sigma_{\mathrm{B}}\right)$, Yield Strength $\left(\sigma_{02}\right)$, and Deformation to Fracture $(\delta)$ on the Deformation Degree after Rolling.

increase in the fraction of high-angle grain boundaries. The parameters of the ultrafine-grained structure formed as a result of such treatment are comparable with aluminum structure parameters after the deformation by other methods of severe plastic deformation.

2. Aluminum microhardness, ultimate strength, and yield strength increase monotonically with increasing degree of deformation by accumulative roll bonding for up to ten rolling cycles, which tells about possible increase in the deformation degree after such treatment for their further increase.

3. The microhardness of aluminum obtained by accumulative roll bonding exceeds the respective value for aluminum after equal-channel angular pressing by $20 \%$ with comparable deformation degrees, which is apparently associated with the strengthening effect of the aluminum oxide particles introduced into the structure during accumulative roll bonding.

4. It was shown in tensile tests that aluminum obtained by accumulative roll bonding manifests strainhardening, i.e. preserve high resistance to deformation localization, after up to ten rolling cycles, which is an important property of this material for its possible practical application.

The work was carried out in the framework of the Basic Research Program of the State Academies of Sciences for 2013-2020. Structural studies by TEM were carried out at the "Nanotech" Shared Use Center (Institute of Strength Physics and Materials Science of Siberian Branch of the Russian Academy of Sciences, Tomsk), and the ones by EBSD analysis were carried out at the "Diagnostics of Nanomaterials' Structure and Properties" Common Use Centre (Belgorod State University, Belgorod).

\section{REFERENCES}

1. R. Z. Valiev and I. V. Alexandrov, Bulk Nanostructured Metallic Materials [in Russian], IKTs "Akademkniga", Moscow (2007).

2. Yu. R. Kolobov, R. Z. Valiev, G. P. Grabovetskaya, et al., Grain-Boundary Diffusion and Properties of Nanostructured Materials [in Russian], Nauka, Novosibirsk (2001).

3. $\quad$ R. Z. Valiev and T. G. Langdon, Prog. Mater. Sc., 51, 881-981 (2006).

4. $\quad$ S. V. Zherebtsov, G. A. Salishchev, R. M. Galeyev, et al., Scripta Mater., 51, 1147-1151 (2004).

5. A. P. Zhilyaev and T. G. Langdon, Prog. Mater. Sci., 53, 893-979 (2008). 
6. Y. Saito, N. Tsuji, H. Utsunomiya, et al., Scripta Mater., 39, 1221-1224 (1998).

7. F. V. Grechnikov, I. P. Popov, and Ya. A. Erisov, Proc. Samara Sci. Cent. Russ. Acad. Sci., 15, No. 4, 165-169 (2013).

8. X. Huang, N. Tsuji, N. Hansen, and Y. Minamino, Mater. Sci. Eng.: A, 340, 265-271 (2003).

9. H. W. Höppel, J. May, and M. Göken, Adv. Eng. Mater., 6, 219-222 (2004).

10. K. V. Ivanov and E. V. Naydenkin, Bull. Tomsk Polytech. Univ., 315, 118-122 (2009).

11. K. V. Ivanov and E. V. Naydenkin, Russ. Phys. J., 52, 1030-1035 (2009).

12. B. L. Li, N. Tsuji, and N. Kamikawa, Mater. Sci. Eng.: A, 423, 331-342 (2006).

13. K. V. Ivanov and E. V. Naydenkin, Mater. Sci. Eng.: A, 606, 313-321 (2014). 